\title{
Comparative hyperthermia effects of silica-gold nanoshells with different surface coverage of gold clusters on epithelial tumor cells
}

This article was published in the following Dove Press journal:

International Journal of Nanomedicine

10 September 2015

Number of times this article has been viewed

\author{
Sang-Eun Park ${ }^{1, *}$ \\ Jaewon Lee ${ }^{2, *}$ \\ Taeksu Lee ${ }^{2}$ \\ Saet-Byeol Bae' \\ Byunghoon Kang ${ }^{2}$ \\ Yong-Min $\mathrm{Huh}^{3}$ \\ Sang-Wha Lee' \\ Seungjoo $\mathrm{Haam}^{2}$
}

'Department of Chemical and Biochemical Engineering, Gachon University, Gyeonggi-Do, Republic of Korea; ${ }^{2}$ Department of Chemical Engineering, Yonsei University, Seoul, Republic of Korea; ${ }^{3}$ Department of Radiology, College of Medicine, Yonsei University, Seoul, Republic of Korea

*These authors contributed equally to this work
Correspondence: Sang-Wha Lee Department of Chemical \& Biochemical Engineering, Gachon University, Seongnam-daero 1342, Sujeong-gu, Seongnam City, Republic of Korea 46I-70I

Email Iswha@gachon.ac.kr

Seungjoo Haam

Department of Chemical Engineering,

Yonsei University, Seoul 120-749,

Republic of Korea

Email haam@yonsei.ac.kr
Abstract: Silica-gold nanoshell (SGNS), which is a silica core surrounded by a gold layer, was synthesized by seed-mediated coalescence of gold clusters in an electroless plating solution. SGNS variations with different surface coverage of gold clusters were prepared by adjusting the amounts of gold salts in the presence of formaldehyde-reducing agents. Fully covered SGNS (f-SGNS) with connected gold clusters exhibited stronger intensity and more redshift of plasmon bands located around $820 \mathrm{~nm}$ than those of partially covered SGNS (p-SGNS) with disconnected gold clusters. Upon irradiation with near-infrared light $\left(30 \mathrm{~W} / \mathrm{cm}^{2}, 700-800 \mathrm{~nm}\right)$, f-SGNS caused a larger hyperthermia effect, generating a large temperature change $\left(\Delta \mathrm{T}=42^{\circ} \mathrm{C}\right)$, as compared to the relatively small temperature change $\left(\Delta \mathrm{T}=24^{\circ} \mathrm{C}\right)$ caused by $\mathrm{p}-\mathrm{SGNS}$. The therapeutic antibody, Erbitux ${ }^{\mathrm{TM}}$ (ERB), was further conjugated to SGNS for specific tumor cell targeting. The f-ERB-SGNS showed excellent therapeutic efficacy based on the combined effect of both the therapeutic antibody and the full hyperthermia dose under near-infrared irradiation. Thus, SGNS with well-controlled surface morphology of gold shells may be applicable for near-infrared-induced hyperthermia therapy with tunable optical properties.

Keywords: gold nanoshell, plasmon resonance, Erbitux, human epithelial cancer, hyperthermia

\section{Introduction}

Recent applications of nanotechnology in medicine have focused on the fabrication of a hybrid core-shell nanostructure, known as a metal nanoshell, consisting of a dielectric core surrounded by a thin metallic layer for precise control of optical resonance that depends on the dielectric constant of the core materials and core-shell ratio. ${ }^{1-4}$ Hybrid nanoshells with tailored optical properties possess the highly tunable plasmon resonance of collective oscillations of free electrons across the visible into the near-infrared (NIR) region, where optical transmittance in the tissue is optimized. Tunable optical properties of nanoshells conjugated with targeting moieties or antibodies can provide noninvasive optical diagnosis and therapeutic payloads such as hyperthermia cancer therapy, photothermal-triggered drug release, and bio-imaging with cell targeting. ${ }^{5-9}$

Because of their biocompatible and/or noncytotoxic properties, gold composites with various dielectric cores such as $\mathrm{Au}_{2} \mathrm{~S}$, Polystyrene latex sphere, hollow sphere, nanomicelles, or silica nanorattles have been actively developed for biological applications. ${ }^{2,10-15}$ Gold nanoshells with a gold sulfide core are formed in a precisely controlled manner, but it is difficult to avoid generating gold nanoparticles (NPs) together with $\mathrm{Au}$-coated $\mathrm{Au}_{2} \mathrm{~S}$ NPs during the fabrication process. ${ }^{10}$ Polystyrene latex spheres with high monodispersity are used as dielectric cores because of their facile 
incorporation of organic dyes. Nonetheless, their plasmon resonances may not produce sufficient radiant local heating because of the low intensity of NIR absorption bands. ${ }^{11}$ A gold cage with strong NIR absorption can be prepared to less than $50 \mathrm{~nm}$ by simply adjusting the processing parameters. ${ }^{12,13}$ Nanomicelles with Au shells are designed to be multifunctional drug-delivery vehicles and to combine bio-imaging, targeting, and light-triggered drug release. ${ }^{14}$

Since the first report ${ }^{1}$ regarding seed-mediated fabrication of silica-gold nanoshell (SGNS) nearly two decades ago, numerous studies ${ }^{2-6,8,13-15}$ have focused on the fabrication of SGNS exhibiting robust structural integrity and the tunable optical resonance by simply adjusting the core-shell ratio across the metal interface, and there have been many attempts to utilize the strong NIR absorption of SGNS as a phototriggered hyperthermia agent on the morbidity of tumor cells. However, there have been concerns regarding the potential human carcinogenicity of silica core materials. ${ }^{16}$ Hirsch et al observed photothermal destruction of carcinoma cells under NIR laser exposure $\left(35 \mathrm{~W} / \mathrm{cm}^{2}\right)$ at $820 \mathrm{~nm}$ for $7 \mathrm{~min}$, and all nanoshell-treated samples underwent photothermal destruction within the laser spot, which was determined by calcein AM viability staining. ${ }^{5}$ Hyeon et al also reported targeted NIR photothermal therapy against cancer cells using magnetic Au nanoshells at $800 \mathrm{~nm}$ under varied laser power densities from $2.5-10 \mathrm{~W} / \mathrm{cm}^{2} .{ }^{17}$ Recently, Au nanorods have been employed for in vitro (or in vivo) photothermal destruction of cancer cells for different $\mathrm{Au}$ nanorods concentrations, NIR laser power densities, and irradiation times. ${ }^{18-20}$ However, the effect of Au shell thickness or surface coverage on the hyperthermiainduced destruction of cancerous cells remains unknown.

Therapeutic antibodies posses to not only specifically bind to corresponding tumor-specific and/or tumor-associated antigens but also kill tumor cells by blocking signaling pathways. ${ }^{21-23}$ Targeted delivery of plasmon-resonant NPs can enhance the selective killing of tumor cells while minimizing the toxicity to neighboring normal cells. One of the most promising targeting moieties for seeking cancerous cells is an antibody capable of specifically binding to tumorspecific antigens. Erbitux (ERB, cetuximab), a monoclonal therapeutic antibody, binds to the extracellular domain of epidermal growth factor receptor (EGFR) to prevent the activation and subsequent dimerization of the receptor, to decrease receptor activation and dimerization and result in inhibition of signal transduction and anti-proliferative effects. ${ }^{24-26}$ This agent may inhibit EGFR-dependent primary tumor growth and metastasis, in which EGFR is overexpressed on the cell surfaces of various solid tumors. Targeted cancer therapeutics using NIR-activated NPs will enable treatment of tumor cells with maximum efficacy while minimizing adverse side effects. In this method, ERB-conjugated nanoshells target human epithelial cancer cells through hyperthermia generated by NIR irradiation. The superficial anatomic location of epithelial cells enables the NIR laser beam to penetrate into tumor cells for photothermal destruction of tumor cells bound to nanoshell particles. ${ }^{27,28}$

In this study, SGNS were prepared by a seed-mediated electroless plating method. Small gold seeds were electrostatically deposited on amine-terminated silica NPs, followed by seed-mediated coalescence of gold clusters. The surface morphology of the Au shell (ie, the surface coverage and thickness of the Au layer) was controlled by adjusting the amounts of Au salts in the presence of formaldehyde reducing agents. Fully covered SGNS (f-SGNS) represent an Au shell with continuous (connected) gold clusters on the surface, while partially covered SGNS (p-SGNS) represent an Au shell with disconnected gold clusters on the surface. The hyperthermia effects on SGNS solution temperature were investigated by using particles with different surface coverage and thickness of Au shell, as well as particle concentration. Furthermore, ERB-conjugated SGNS with different surface coverages of $\mathrm{Au}$ clusters were examined for their ability to target and be taken up by human epithelial cancer cells, which overexpress the EGFR tumor antigen. We also report the killing effect of epithelial cancer cells through synergistic blocking of the signaling pathway by the therapeutic antibody and hyperthermia using a NIR laser.

\section{Materials and methods Chemical materials and analysis}

3-Aminopropyl trimethoxysilane (97\%), tetraethyl orthosilicate (99.999\%), tetrakis(hydroxymethyl) phosphonium chloride (80\% solution), ammonium hydroxide (30\%), formaldehyde $(\mathrm{HCOH}, 37 \%)$, L-ascorbic acid $\left(\mathrm{C}_{6} \mathrm{H}_{8} \mathrm{O}_{6}\right.$, $99 \%)$, hydrogen tetra-chloroaurate (III) hydrate $\left(\mathrm{HAuCl}_{4}\right.$, $99.99 \%)$, potassium carbonate $\left(\mathrm{K}_{2} \mathrm{CO}_{3}, 99.7 \%\right)$, absolute ethanol (ETOH, 99.5\%), and $\mathrm{NaOH}$ (semiconductor grade) were purchased from Sigma-Aldrich (St Louis, MO, USA) and used as received.

UV-Visible spectra were collected using an HP8453 UVVisible spectrometer (Agilent Technologies, Santa Clara, CA, USA) in the range of 200-1100 nm. All samples were dispersed in water and loaded into a quartz cell for analysis. High-resolution scanning electron microscope images were obtained using a cold-type field emission gun scanning electron microscope operating at $15 \mathrm{kV}$ accelerating voltage. 
The sample was placed and pressed onto double-sided conducting carbon tape supported by a copper plate. The X-ray diffraction patterns of the elemental composition were measured using a Rigaku D/MAX-2200 of $3 \mathrm{~kW}$ X-ray generator (using $\mathrm{Cu} \mathrm{K} \alpha=1.5418$ radiation) (Tokyo, Japan).

\section{Synthesis of SGNS}

The synthetic procedures were described in detail elsewhere. ${ }^{29,30}$ Colloidal silica were prepared using the Stöber method, which is based on the simultaneous hydrolysis and condensation of tetraethyl ortho-silicate by an ammonium hydroxide catalyst. ${ }^{31}$ Gold colloids (1-3 nm) were prepared by mixing $2.0 \mathrm{~mL}$ of $1.0 \mathrm{wt} \%$ tetrachloroaurate (III) trihydrate, $0.5 \mathrm{~mL}$ of $1.0 \mathrm{M}$ $\mathrm{NaOH}$, and $1.0 \mathrm{~mL}$ of $50 \mathrm{mM}$ aqueous tetrakis (hydroxymethyl) phosphonium chloride, which is a powerfully reducing agent that can reduce gold salts via the derivation of formaldehyde. ${ }^{32}$ Next, the surface of silica NPs was functionalized using aminopropyltrimethoxy silane with a terminal amine group. Small Au seeds with negative charge were electrostatically attached onto the amine-terminated silica NPs. The Au seeds on the silica surface were grown by reducing the $\mathrm{Au}$ salts in the presence of formaldehyde reducing agents (80 to $120 \mu \mathrm{L}$ ), producing an Au layer. Au coverage on the silica NPs was adjusted by changing the concentration of reducible Au salts during the seed-mediated growth of Au clusters. The fabrication procedures are summarized in Figure 1.

\section{Cellular viability test of ERB-conjugated SGNS}

The cytotoxic effect of ERB-conjugated SGNS in A431 and MCF7 cells was analyzed using a colorimetric assay based on the mitochondrial oxidation of 3-(4,5-dimethylthiazolyl-2)2,5-diphenyltetrazolium bromide (MTT). A431 and MCF7 cells were maintained in Dulbecco's Modified Eagle's Medium (DMEM) containing 10\% fetal bovine serum (FBS) and $1 \%$ antibiotics at $37^{\circ} \mathrm{C}$ under a $5 \% \mathrm{CO}_{2}$ atmosphere. The cells were diluted to a density of $1.0 \times 10^{4}$ cells $/ 100 \mu \mathrm{L}$ in a 96-well plate and incubated at $37^{\circ} \mathrm{C}$ at $5 \% \mathrm{CO}_{2}$. A431 and MCF7 cells were treated with various concentrations of ERB-conjugated SGNS $\left(5.12 \times 10^{-5} \mathrm{mg} / \mathrm{L}, \sim 20 \mathrm{mg} / \mathrm{L}\right)$ overnight and rinsed with $100 \mu \mathrm{L}$ phosphate-buffered saline (pH 7.4, $1 \mathrm{mM}$ ), after which freshly-prepared MTT solution was added. The cells were further incubated for $4 \mathrm{~h}$, and then $100 \mu \mathrm{L}$ of solubilization solution ( $10 \%$ sodium dodecyl sulfate in $0.01 \mathrm{M} \mathrm{HCl}$ ) was added. After overnight incubation, the absorbance of each well was measured using an enzymelinked immunosorbent assay plate reader (Spectra MAX 340, Molecular Devices, Sunnyvale, CA, USA) at an absorbance wavelength of $575 \mathrm{~nm}$ and a reference wavelength of $650 \mathrm{~nm}$. Cell viabilities were obtained by calculating the ratio of the intensity of purple formazan in viable cells treated with ERBconjugated SGNS to the intensity in untreated control cells. This research is performed under the highest ethical and legal standards with the permission from the Ethics Committee of the Korean Biochip Society for the acquisition of human cells, cellular toxicity and photothermal assay tests.

\section{In vitro photothermal experiments of ERB-conjugated SGNS}

The epidermoid carcinoma A431 cell line and MCF7 cell line (with different levels of EGFR over-expression) were obtained from the American Tissue Type Culture (ATCC, Manassas, VA, USA) and cultured. Briefly, the cells $\left(1 \times 10^{6}\right.$ cells $\left./ \mathrm{mL}\right)$ were seeded in $100 \varnothing$ Corning culture dishes (10 mL/dish; Corning, NY, USA) with DMEM supplemented with $10 \% \mathrm{FBS}$ and $1 \%$ antibiotics and incubated at $37^{\circ} \mathrm{C}$ in

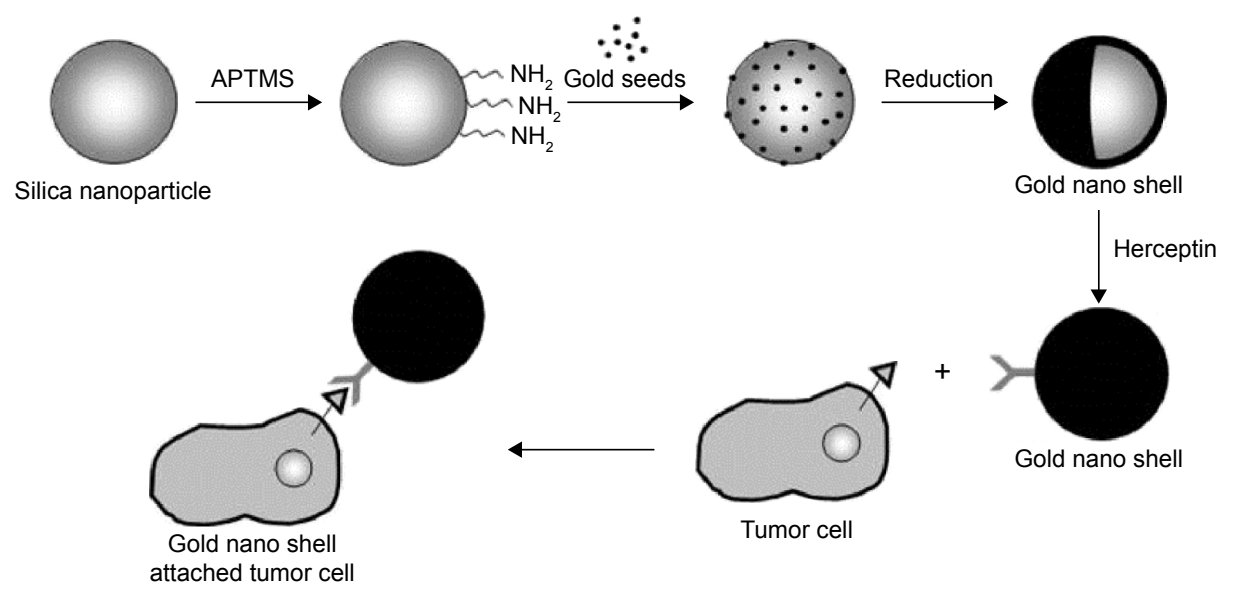

Figure I Conceptual scheme of SGNS for tumor-specific targeting. Abbreviations: SGNS, silica-gold nanoshell; APTMS, (3-Aminopropyl)trimethoxysilane. 
a humidified atmosphere containing $5 \% \mathrm{CO}_{2}$. The A431 and MCF7 cell lines $\left(1 \times 10^{4}\right.$ cells/well $)$ were incubated in 96-well plates for $24 \mathrm{~h}$. The cells were incubated with ERB-SGNS $\left(1.6 \times 10^{10}\right.$ particles $\left./ \mathrm{mL}\right)$ at $37^{\circ} \mathrm{C}$. After $1 \mathrm{~h}$, free SGNS were removed by washing with an equal volume of $10 \% \mathrm{FBS}$. The cells treated with SGNS were then incubated within DMEM supplemented with $10 \% \mathrm{FBS}$ and $1 \%$ antibiotics at $37^{\circ} \mathrm{C}$.

After exposure to an NIR laser at $820 \mathrm{~nm}\left(35 \mathrm{~W} / \mathrm{cm}^{2}\right.$ for $5 \mathrm{~min}$ ), the cells were further incubated for an additional $2 \mathrm{~h}$ at $37^{\circ} \mathrm{C}$. Cell viability was determined using staining calcein AM (Molecular Probes, $1 \mu \mathrm{M}$ ) and by measuring the emission after excitation at $488 \mathrm{~nm}$ using a fluorescence spectrometer reader. The live/dead cell distribution after calcein AM staining was observed using a fluorescence microscope.

\section{Results and discussion}

SGNS with different surface coverage of Au clusters were prepared..$^{30}$ The surface coverage of Au clusters over the silica NPs was controlled by adjusting the concentration of reducible $\mathrm{Au}$ salts relative to the number of silica NPs $\left(1.5 \times 10^{13} / \mathrm{cm}^{3}\right){ }^{2}$ As shown in Figure 2, SGNS prepared with $0.38 \mathrm{mM}$ of Au salts exhibited a rough surface morphology consisting of partial coverage of gold clusters, while SGNS prepared with $1.52 \mathrm{mM}$ of gold salts exhibited a relatively smooth surface morphology with full coverage of Au clusters. Excessive addition of $\mathrm{Au}$ salts resulted in a significant reduction in plasmon bands due to the aggregation of gold NPs (data not shown). ${ }^{29}$

Figure 3 shows the X-ray diffraction patterns of SGNS with different surface coverage of Au clusters. The sample solution was dropped onto a $\mathrm{SnO}_{2}$ glass more than 50 times to prepare a multi-layered deposition of SGNS. f-SGNS prepared with $1.52 \mathrm{mM}$ Au salts exhibited a distinct X-ray diffraction peak attributed to the crystalline $\mathrm{Au}$ (111) phase. On the other hand, p-SGNS prepared by $0.38 \mathrm{mM}$ gold salts exhibited a very similar X-ray diffraction pattern to Au seeddeposited silica NPs $\left(\mathrm{Au}-\mathrm{SiO}_{2}\right)$, and the amorphous silica layer was distinctly observed in both samples (p-SGNS and $\mathrm{Au}-\mathrm{SiO}_{2}$ ), indicating significant exposure of the bare silica surface. The growth of deposited Au seeds did not produce full coverage of Au layer over silica cores when the Au salts were not sufficiently available.

$\mathrm{Au}-\mathrm{SiO}_{2}$ NPs showed a characteristic absorption peak at $520 \mathrm{~nm}$, corresponding to the $\mathrm{Au}$ seeds deposited on the silica NPs (data not shown). According to Figure 4, plasmon-derived absorption bands (plasmon bands) of SGNS ranged from $700-820 \mathrm{~nm}$, depending on the surface coverage of $\mathrm{Au}$ clusters over silica cores. ${ }^{29,33}$ A strong plasmon band with a significant redshift was observed by f-SGNS, whereas p-SGNS exhibited a weak plasmon band with a less significant redshift. It is generally known that plasmonderived absorption bands are strongly enhanced by plasmon couplings between adjacent $\mathrm{Au}$ clusters. ${ }^{34}$

In contrast, antibody-conjugated f-SGNS exhibited much lower optical intensity compared to bare f-SGNS. This may be because the entire surface of f-SGNS was conjugated with ERB antibodies, which induced a change in the dielectric environment and the agglomeration of SGNS via ERB linkage. However, ERB-bound p-SGNS (p-ERBSGNS) exhibited a slightly more intense absorption peak compared to that of bare $\mathrm{p}-\mathrm{SGNS}$. The p-SGNS appeared to have fewer conjugation sites for the ERB antibody, leading to less inhibitive action for optical absorption of SGNS, and the conjugation of ERB was not effective on p-SGNS. ${ }^{24,26}$

The surface plasmon wave was propagated along the interface between the silica cores and Au shell layers, leading to the collective oscillation of plasmon electrons which can induce Au-layer heating, known as the hyperthermia effect.
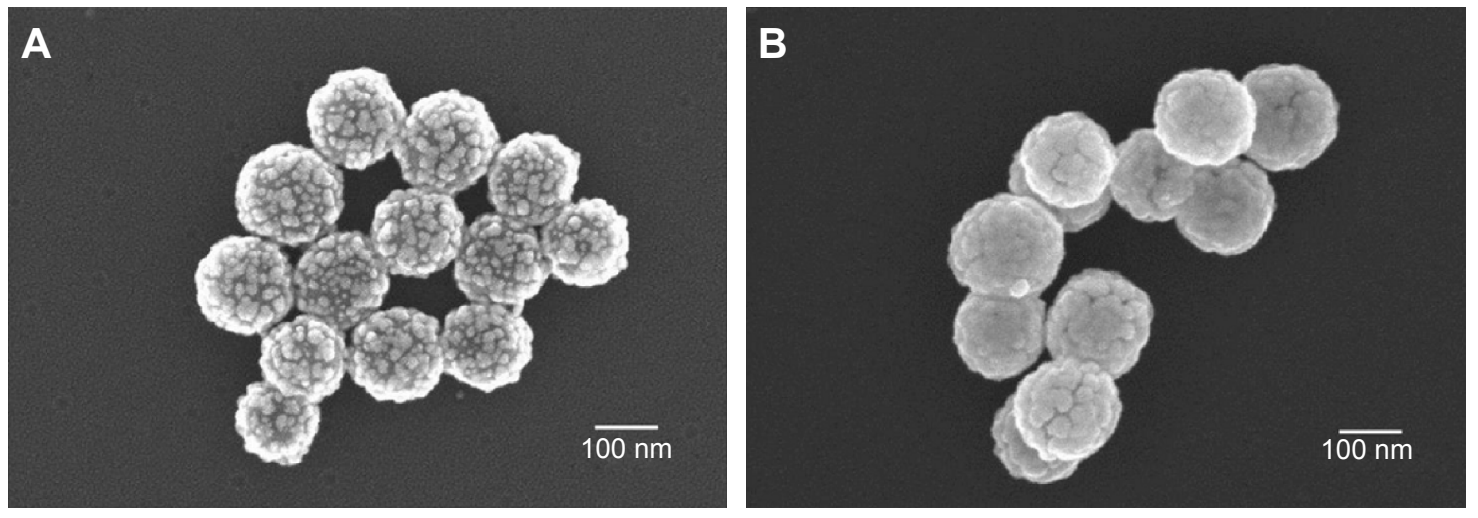

Figure 2 Scanning electron microscope images of SGNS (Silica core $=120 \mathrm{~nm}$ ) prepared by adding $80 \mu \mathrm{L}$ of $\mathrm{HCHO}$ reducing agent and different amounts of Au salts: (A) $0.38 \mathrm{mM}$ of Au salts, (B) $1.52 \mathrm{mM}$ of Au salts.

Abbreviation: SGNS, silica-gold nanoshell. 


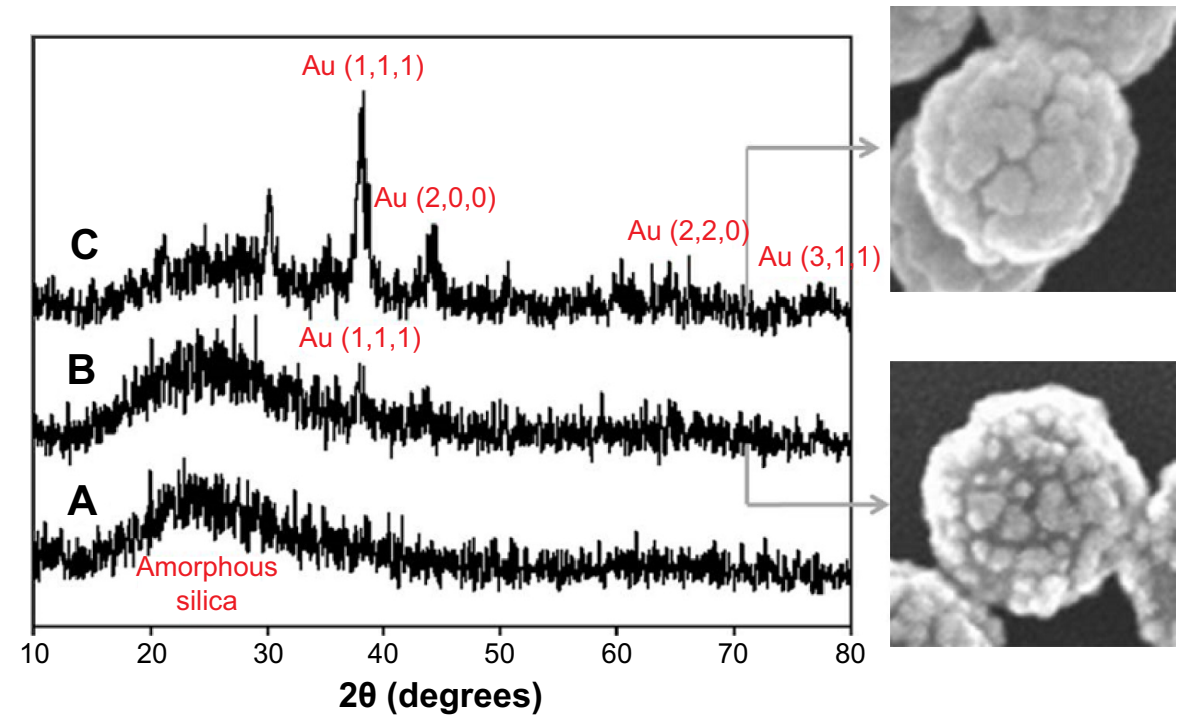

Figure 3 X-ray diffraction analysis of SGNS: (A) Au-deposited silica, (B) SGNS prepared with 0.38 mM of Au salts, (C) SGNS prepared with I.52 mM of Au salts. The upper and lower inserts are the magnified SEM images of f-SGNS and p-SGNS, respectively.

Abbreviations: SGNS, silica-gold nanoshell; p-SGNS, partially covered-silica-gold nanoshell; f-SGNS, fully covered-silica-gold nanoshell.

To investigate the photothermal response of p-SGNS and f-SGNS under NIR light irradiation, we used an NIR laser $\left(30 \mathrm{~W} / \mathrm{cm}^{2}\right)$ at fixed wavelengths of $700 \mathrm{~nm}$ and $800 \mathrm{~nm}$, corresponding to p-SGNS and f-SGNS, respectively. Figure 5A shows the temperature changes of the sample solution with increasing NIR irradiation times. The final temperature of the p-SGNS and f-SGNS solutions asymptotically approached $49^{\circ} \mathrm{C}$ and $67^{\circ} \mathrm{C}$ after exposure to the NIR laser for $800 \mathrm{sec}$. No significant temperature change was observed in pure water and $\mathrm{Au}-\mathrm{SiO}_{2}\left(\Delta \mathrm{T}<10^{\circ} \mathrm{C}\right)$ under the same irradiation

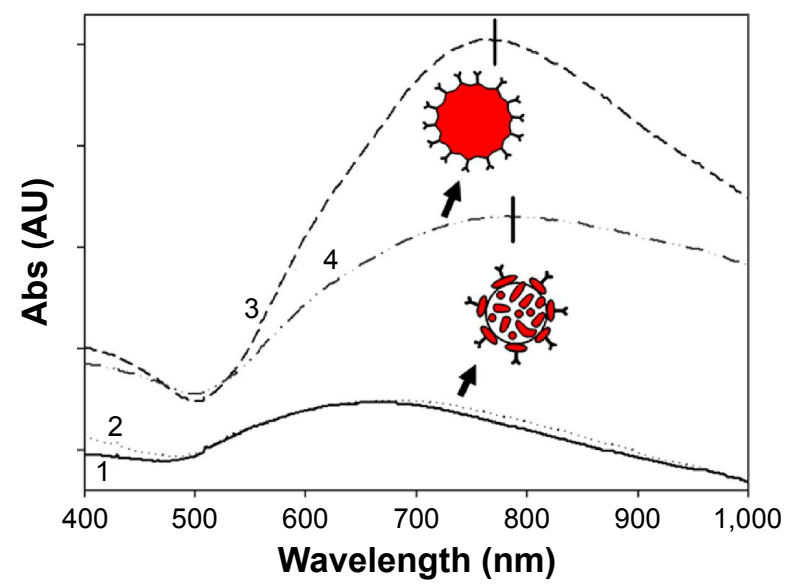

Figure 4 UV-Vis spectra of SGNS with partially and fully-covered Au clusters before and after ERB conjugation. The vertical lines indicate the maximum peak position. The arrows point out the UV-vis spectra of corresponding nanoshells. Notes: Spectra numbers of I and 2 correspond to p-SGNS before and after conjugation to ERB. Numbers 3 and 4 indicate f-SGNS before and after conjugation to ERB. Abbreviations: SGNS, silica-gold nanoshell; p-SGNS, partially covered-silica-gold nanoshell; f-SGNS, fully covered-silica-gold nanoshell; UV-Vis, UV-Visible spectroscopy; ERB, Erbitux ${ }^{\mathrm{TM}}$; Abs, absorption. conditions. Thus, the conversion efficiency of NIR light to thermal energy was higher for f-SGNS with full coverage of the Au layer compared to that of p-SGNS with partial coverage of the Au clusters.

Temperature change of the sample solution under NIR irradiation was also dependent on the particle concentration. Over an elapsed time of $180 \mathrm{sec}$, solution temperature changes were investigated by diluting the original SGNS solution at room temperature. As shown in Figure 5B, the temperature change was proportional to the number of SGNS particles in the solution. At an elapsed time of $180 \mathrm{sec}$, the 10 -fold diluted sample $\left(0.15 \times 10^{13} / \mathrm{mL}\right)$ had a final temperature of approximately $35^{\circ} \mathrm{C}$, while 5 -fold diluted sample $\left(0.30 \times 10^{13} / \mathrm{mL}\right)$ had a final temperature of $>40^{\circ} \mathrm{C}$. The two-fold diluted sample $\left(0.75 \times 10^{13} / \mathrm{mL}\right)$ and original sample $\left(1.5 \times 10^{13} / \mathrm{mL}\right)$ showed similar solution temperature changes until $60 \mathrm{sec}$, after which the original sample showed a more rapid increase in solution temperature compared to the 2 -fold diluted sample. The final temperature difference between the original sample and the 2-fold diluted sample was approximately $5^{\circ} \mathrm{C}$. When more than $0.75 \times 10^{13} / \mathrm{mL}$ particles were used, a temperature change was not observed, likely because of the rapid thermal loss to the ambient environment.

Additionally, we investigated the hyperthermia effect of SGNS on the solution temperature depending on the Au shell thickness under NIR irradiation $\left(820 \mathrm{~nm}, 30 \mathrm{~W} / \mathrm{cm}^{2}\right.$ for $\left.3 \mathrm{~min}\right)$. According to Figure S1 and S2, SGNS with the thickest $\mathrm{Au}$ shell (Au shell thickness $=35 \mathrm{~nm})$ and the strongest absorption band showed a lower effective hyperthermia effect; the SGNS (Au shell $=35 \mathrm{~nm}$ ) exhibited a lower increase 
A

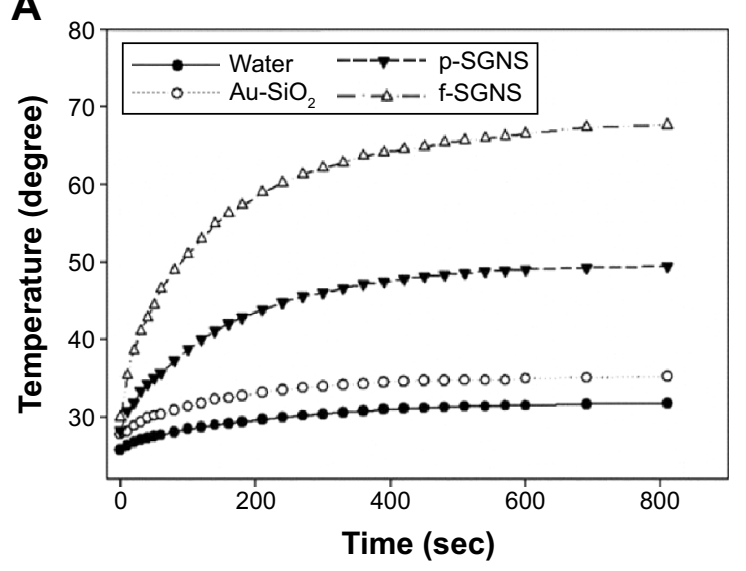

B

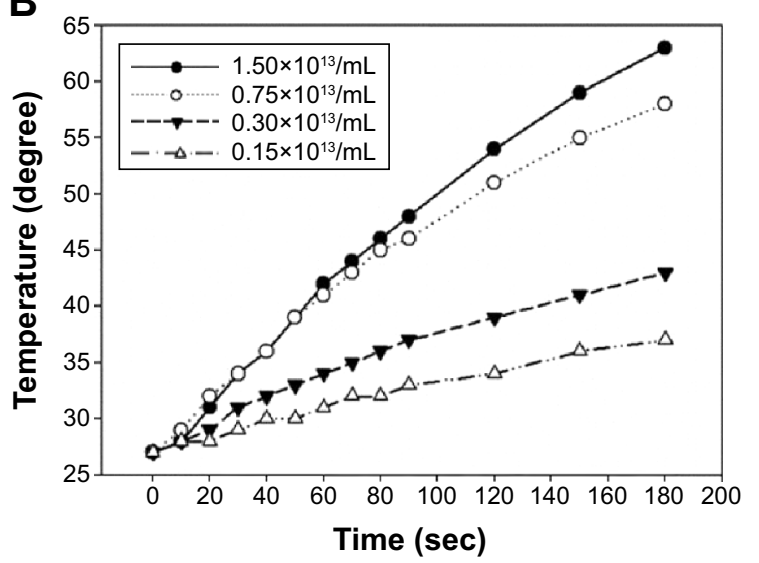

Figure $\mathbf{5}$ Temperature change of SGNS solution with increasing exposure times of NIR irradiation: (A) as-prepared samples with different surface coverage of Au clusters, including pure water, $($ B $)$ different dilution ratio $(\times 1, \times 2, \times 5, \times 10)$ of original SGNS solution $\left(1.5 \times 10^{13} / \mathrm{mL}\right)$.

Abbreviations: SGNS, silica-gold nanoshell; p-SGNS, partially covered-silica-gold nanoshell; f-SGNS, fully covered-silica-gold nanoshell; sec, seconds; NIR, near infra-red.

in solution temperature compared to the change for SGNS (Au shell thickness $=20,28 \mathrm{~nm}$ ) with lower absorption bands. The hyperthermia effect is mainly attributed to the plasmon resonances occurring at the interface between the Au layer and the dielectric silica core. Thus, a thick Au layer may be less effective for generating a full hyperthermia effect at the interface because the absorbed light is prone to dissipation within the thick Au layer via bulk plasmon, leading to smaller increases in the solution temperature. ${ }^{35}$ In this work, an optimal thickness of the Au layer achieved an efficient hyperthermia effect, but disconnected Au clusters or a thick Au layer was not appropriate as the efficient hyperthermia agent.

Under NIR irradiation with $35 \mathrm{~W} / \mathrm{cm}^{2}$ and for a spot diameter of $2 \mathrm{~mm}$, cell death was observed on both p-ERBSGNS- and f-ERB-SGNS-treated samples at $700 \mathrm{~nm}$ and
$800 \mathrm{~nm}$, respectively. Under control conditions (no nanoshell or nonspecific antibody), no cell death was observed, as shown in Figure 6. In vitro studies at high laser intensity indicated that the surface coverage of Au clusters had no significant effect on the photothermal triggered damage of tumor cells treated with p-ERB-SGNS and f-ERB-SGNS. Specifically designed photothermal nanomaterials have attracted attention because of their efficacy in treatment and because targeted NIR laser therapy does not require surgical intervention. Thus, the hybrid core-shell nanostructures, composed of metal nanoshells consisting of a dielectric core and metallic layer, are considered to be suitable photothermal probes in nanomedicine because of their highly tuneable surface plasmon resonance across the visible light into NIR region where optical transmittance in the tissue is optimized..$^{2,3}$

\section{A}

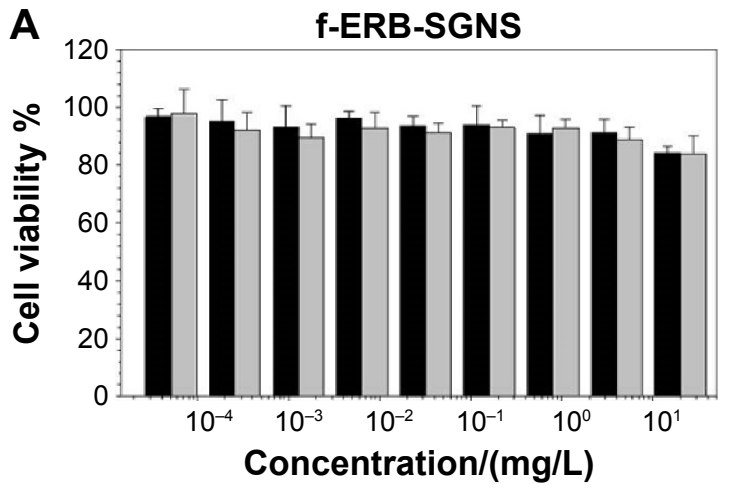

B

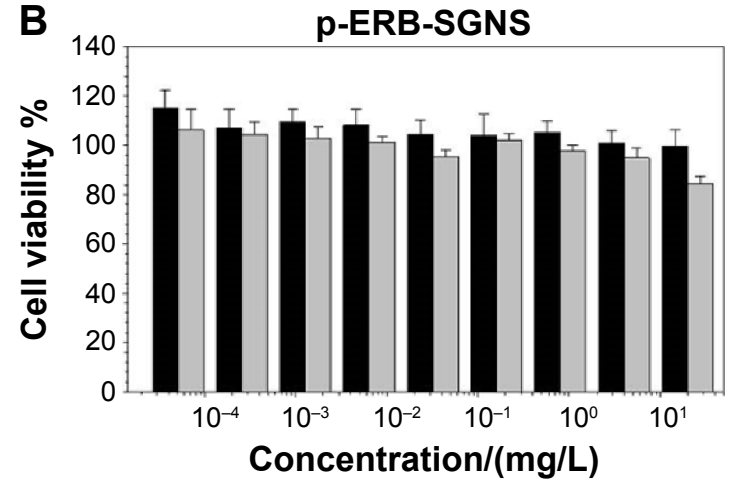

$$
\text { A431 } \square \text { MCF7 }
$$

Figure 6 Cell viability test of ERB-conjugated SGNS treated A43I (dark bar graph) and MCF7 cells (gray bar graph) (I $\times 10^{4}$ cells each) with various dosages of (A) f-ERBSGNS and (B) P-ERB-SGNS $\left(5.12 \times 10^{-5}, \sim 20 \mathrm{mg} / \mathrm{L}\right)$ using the MTT assay at $37^{\circ} \mathrm{C}$ under a $5 \% \mathrm{CO}_{2}$ atmosphere after incubation overnight.

Abbreviations: SGNS, silica-gold nanoshell; ERB, Erbitux ${ }^{\mathrm{TM}}$; p-ERB-SGNS, ERB-conjugated partially covered SGNS; f-ERB-SGNS, ERB-conjugated fully covered SGNS; MTT, 3-(4,5-dimethylthiazolyl-2)-2,5-diphenyltetrazolium bromide. 
Prior to photothermal ablation experiments using SGNS, we performed an MTT assay to measure the cytotoxicity of p-ERB-SGNS and f-ERB-SGNS. The target cells (A431 cells over-expressing EGFR and MCF7 cells lowly expressing EGFR) were treated with various concentrations of p-ERBSGNS and f-ERB-SGNS $\left(5.12 \times 10^{-5} \mathrm{mg} / \mathrm{L}, \sim 20 \mathrm{mg} / \mathrm{L}\right)$ overnight and cell viabilities were analyzed (Figure 6 ). The results showed that cellular viability was higher than $80 \%$ in the presence of p-ERB-SGNS and f-ERB-SGNS, indicating low cytotoxicity within this concentration range.

To determine the turmoricidal efficacies of NIR laser treatment modalities, cancer cells were incubated with the prepared NPs after laser exposure. The therapeutic efficacies of p-ERB-SGNS and f-ERB-SGNS were then examined with respect to laser intensity and the concentration of SGNS against the cancer cell lines A431 and MCF7 cells with different EGFR overexpression levels. The viability of MCF7 and A431 cells was not influenced by the intensity of
NIR-laser only (Figure 7, control). The f-ERB-SGNS- and p-ERB-SGNS-treated MCF7 cells showed strong emission after calcein AM staining. Calcein AM does not dye dead cells; this demonstrated that MCF7 cells did not heal by hyperthermia because of low EGFR receptor level. However, A431 cells had high EGFR receptor levels. Therefore, f-ERB-SGNS- and p-ERB-SGNS-treated A431 cells emitted weak light because of the hyperthermia effect from NIR-absorbing nanoshells (such as f-SGNS and p-SGNS). Particularly, we quantified cell viabilities after treatment with $0.4 \mathrm{mg} / \mathrm{L}$ of p-ERG-SGNS and $0.4 \mathrm{mg} / \mathrm{L}$ of f-ERB-SGNS by measuring the relative fluorescence intensities. The cellular viability of the control group was considered to be $100 \%$. Cell viability after f-ERB-SGNS treatment of A431 cells was $20.57 \% \pm 0.23 \%$ which was lower than that of p-ERBSGNS $(45.25 \% \pm 1.5 \%)$. The therapeutic efficacy of f-SGNS was confirmed to be better than that of p-SGNS, which was expected based on the strong NIR absorbance of f-SGNS.

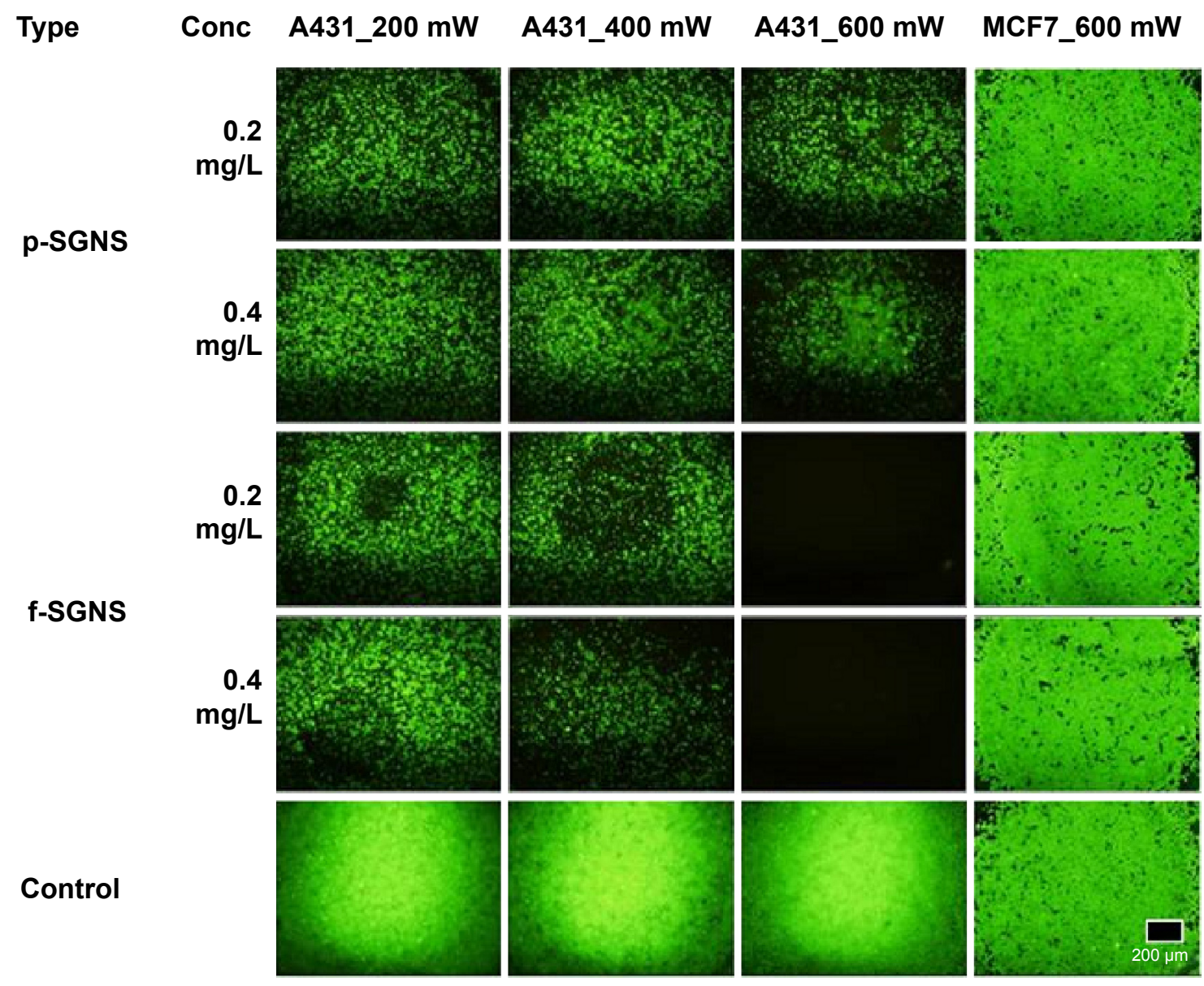

Figure 7 Combined imaging and therapy of epidermoid carcinoma A43I cell line and MCF7 cell line (with different levels of EGFR overexpression). Cytotoxicity of control samples was tested using cells treated with an NIR-emitting laser only. Images of cells (A43I, MCF7) targeted with ERB-SGNS were obtained upon exposure to increasing laser intensity $(200,400,600 \mathrm{~mW})$. Cell viabilities were estimated by calcein staining (Molecular Probes, I $\mu \mathrm{M}$ ).

Abbreviations: SGNS, silica-gold nanoshell; P-SGNS, partially covered-silica-gold nanoshell; f-SGNS, fully covered-silica-gold nanoshell; Conc, concentration; EGFR, epidermal growth factor receptor; NIR, near infra-red; ERB, Erbitux ${ }^{\mathrm{TM}}$; p-ERB-SGNS, ERB-conjugated partially covered SGNS; f-ERB-SGNS, ERB-conjugated fully covered SGNS; MTT, 3-(4,5-dimethylthiazolyl-2)-2,5-diphenyltetrazolium bromide. 
Additionally, both p-ERB-SGNS- and f-ERB-SGNS-treated MCF7 cells showed relatively high cell viabilities $(0.4 \mathrm{mg} / \mathrm{L}$ of p-SGNS-treated MCF7 cells: $98.95 \% \pm 0.4 \%$ and $0.4 \mathrm{mg} / \mathrm{L}$ of f-SGNS-treated MCF7 cells: $98.95 \% \pm 0.8 \%$ ), indicating that there was no photothermal therapeutic effect on MCF7 cells caused by the antibody (ERB) on NP surface. Thus, we evaluated the increased therapeutic efficacy by f-ERB-SGNS under high-intensity NIR irradiation.

In summary, we examined the use of the therapeutic antibody ERB-conjugated SGNS for photothermal therapy of epithelial cancer cells. The surface morphology of the $\mathrm{Au}$ shell (surface coverage and thickness of the Au layer) was controlled by changing the relative amounts of $\mathrm{Au}$ precursors and reducing agents. Au layer with full surface coverage and optimal thickness is confirmed to induce the efficient hyperthermia effect of SGNS, whereas disconnected $\mathrm{Au}$ clusters or a thick $\mathrm{Au}$ layer is not recommended as an efficient hyperthermia agent. In addition, ERB on SGNS not only minimized the toxicities to neighboring normal cells, but also killed tumor cells by blocking signaling pathways. ${ }^{21,26}$

\section{Conclusion}

Silica-gold nanoshell (SGNS) was synthesized using seed-mediated growth into a clustered Au layer in an electroless plating solution. SGNS with different surface morphology of gold layer clusters was prepared by adjusting the amounts of gold salts and reducing agents. The hyperthermia effect of SGNS was systematically compared by using particles with different surface coverage and thickness of the Au layer. The f-SGNS showed more redshift and stronger absorption bands compared to those of p-SGNS. Upon irradiation with NIR light (30 W/cm², 700-800 nm), f-SGNS exhibited higher efficiency of the hyperthermia effect $\left(\Delta \mathrm{T}=42^{\circ} \mathrm{C}\right)$ compared to p-SGNS $\left(\Delta \mathrm{T}=24^{\circ} \mathrm{C}\right)$. Furthermore, ERB-conjugated f-SGNS selectively recognized target cancer cell lines and showed excellent therapeutic efficacy because of the therapeutic antibody and NIR laserinduced hyperthermia effect.

\section{Acknowledgments}

This work was supported by the GRRC program of Gyeonggi province (GRRC Gachon 2014-B04, Development of Nanomaterials for Biomedical Sensing Applications) and was also supported by a grant (15172MFDS241) from Ministry of Food and Drug Safety in 2015.

\section{Disclosure}

The authors report no conflicts of interest in this work.

\section{References}

1. Westcott SL, Oldenburg SJ, Lee TR, Halas NJ. Formation and adsorption of clusters of gold nanoparticles onto functionalized silica nanoparticle surfaces. Langmuir. 1998;14:5396-5401.

2. Pham T, Jackson JB, Halas NJ, Lee TR. Preparation and characterization of gold nanoshells coated with self-assembled monolayers. Langmuir. 2002;18:4915-4920.

3. Prodan E, Radloff C, Halas NJ, Nordlander P. A hybridization model for the plasmon response of complex nanostructures. Science. 2003; 302(5644):419-422.

4. Sauerbeck C, Haderlein M, Schürer B, Braunschweig B, Peukert W, Klupp Taylor RN. Shedding light on the growth of gold nanoshells. ACS Nano. 2014;8(3):3088-3396.

5. Hirsch LR1, Stafford RJ, Bankson JA, et al. Nanoshell-mediated nearinfrared thermal therapy of tumors under magnetic resonance guidance. Proc Natl Acad Sci U S A. 2003;100(23):13549-13554.

6. Hirsch LR, Jackson JB, Lee A, Halas NJ, West JL. A whole blood immunoassay using gold nanoshells. Anal Chem. 2003;75(10): 2377-2381.

7. Yang X, Skrabalak SE, Li ZY, Xia Y, Wang LV. Photoacoustic tomography of a rat cerebral cortex in vivo with au nanocages as an optical contrast agent. Nano Lett. 2007;7(12):3798-3802.

8. Loo C, Lin A, Hirsch L, et al. Nanoshell-enabled photonics-based imaging and therapy of cancer. Technol Cancer Res Treat. 2004;3(1): $33-40$.

9. Dykman L, Khlebtsov N. Gold nanoparticles in biomedical applications: recent advances and perspectives. Chem Soc Rev. 2012;41(6): 2256-2282.

10. Averitt R, Sarkar D, Halas N. Plasmon resonance shifts of Au-coated $\mathrm{Au} 2 \mathrm{~S}$ nanoshells: insight into multicomponent nanoparticle growth. Phys Rev Lett. 1997;78:4217-4220.

11. Shi W, Sahoo Y, Swihart MT, Prasad PN. Gold nanoshells on polystyrene cores for control of surface plasmon resonance. Langmuir. 2005; 21(4):1610-167.

12. Chen JJ, Saeki F, Wiley BJ, et al. Gold nanocages: bioconjugation and their potential use as optical imaging contrast agents. Nano Lett. 2005;5(3):473-47.

13. Liu Z, Song H, Yu L, Yang L. Fabrication and near-infrared photothermal conversion characteristics of Au nanoshells. Applied Physics Letters. 2005;86:113109.

14. Ma Y, Liang X, Tong S, Bao G, Ren Q, Dai Z. Gold Nanoshell Nanomicelles for Potential Magnetic Resonance Imaging, Light-Triggered Drug Release, and Photothermal Therapy. Advanced Functional Materials. 2013;23:815-822.

15. Liu H, Chen D, Li L, et al. Multifunctional Gold Nanoshells on Silica Nanorattles: A Platform for the Combination of Photothermal Therapy and Chemotherapy with Low Systemic Toxicity. Angewandte Chemie. 2011;123:921-925.

16. Jain PK, Lee KS, El-Sayed IH, El-Sayed MA. Calculated absorption and scattering properties of gold nanoparticles of different size, shape, and composition: applications in biological imaging and biomedicine. The Journal of Physical Chemistry B. 2006;110:7238-7248.

17. Kim J, Kim HS, Lee N, Kim T, Kim H, Yu T, Song IC, Moon WK, Hyeon T. Multifunctional uniform nanoparticles composed of a magnetite nanocrystal core and a mesoporous silica shell for magnetic resonance and fluorescence imaging and for drug delivery. Angewandte Chemie. 2008;47:8438-8441.

18. Lin J-T, Chiang Y-S, Lin G-H, Lee H, Liu H-W. In vitro Photothermal Destruction of Cancer Cells Using Gold Nanorods and Pulsed-Train Near-Infrared Laser. Journal of Nanomaterials. 2012:1-6.

19. Hong Y, Lee E, Choi J, et al. Gold Nanorod-Mediated Photothermal Modulation for Localized Ablation of Cancer Cells. Journal of Nanomaterials. 2012:1-7.

20. Popp MK, Oubou I, Shepherd C, Nager Z, Anderson C, Pagliaro L. Photothermal Therapy Using Gold Nanorods and Near-Infrared Light in a Murine Melanoma Model Increases Survival and Decreases Tumor Volume. Journal of Nanomaterials. 2014:1-8. 
21. Clynes RA, Towers TL, Presta LG, Ravetch JV. Inhibitory Fc receptors modulate in vivo cytotoxicity against tumor targets. Nature Medicine. 2000;6:443-446.

22. Yarden Y, Sliwkowski MX. Untangling the ErbB signalling network, Nature reviews. Molecular Cell Biology. 2001;2:127-137.

23. Ridgway J, Zhang G, Wu Y, et al. Inhibition of Dll4 signalling inhibits tumour growth by deregulating angiogenesis. Nature. 2006;444: 1083-1087.

24. Harari PM, Huang S. Radiation combined with EGFR signal inhibitors: head and neck cancer focus. Seminars in Radiation Oncology. 2006;16: 38-44.

25. Dassonville O, Bozec A, Fischel JL, Milano G. EGFR targeting therapies: monoclonal antibodies versus tyrosine kinase inhibitors. Similarities and differences. Critical Reviews in Oncology/Hematology. 2007;62:53-61.

26. Baselga J. The EGFR as a target for anticancer therapy - focus on cetuximab. European Journal of Cancer. 2001;37:16-22.

27. Choi J, Yang J, Park J, et al. Specific Near-IR Absorption Imaging of Glioblastomas Using Integrin-Targeting Gold Nanorods. Advanced Functional Materials. 2011;21:1082-1088.

28. Lee T, Bang D, Park Y, et al. Gadolinium-enriched polyaniline particles (GPAPs) for simultaneous diagnostic imaging and localized photothermal therapy of epithelial cancer. Advanced Healthcare Materials. 2014;3: 1408-1414.
29. Park S, Park M, Han P, Lee S. Relative Contributions of Experimental Parameters to NIR-Absorption Spectra of Gold Nanoshells. J Ind Eng Chem. 2007;13:65-70.

30. Park S-E, Park M-Y, Han P-K, Lee S-W. The Effect of pH-adjusted Gold Colloids on the Formation of Gold Clusters over APTMS-coated Silica Cores. Bulletin of the Korean Chemical Society. 2006;27: 1341-1345.

31. Stöber W, Fink A, Bohn E. Controlled growth of monodisperse silica spheres in the micron size range. Journal of Colloid and Interface Science. 1968;26:62-69.

32. Duff DG, Baiker A, Edwards PP. A new hydrosol of gold clusters. 1. Formation and particle size variation. Langmuir. 1993;9: 2301-2309.

33. Park S-E, Lee J-W, Haam S-J, Lee S-W. Fabrication of double-doped magnetic silica nanospheres and deposition of thin gold layer. Bull Korean Chem Soc. 2009;30:869.

34. Xia Y, Halas NJ. Shape-Controlled Synthesis and Surface Plasmonic Properties of Metallic Nanostructures. MRS Bulletin. 2011;30: 338-348.

35. Raether H. Surface Plasmons on smooth and rough surfaces and on gratings. Springer-Verag, New York, 1986. 


\section{Supplementary materials}
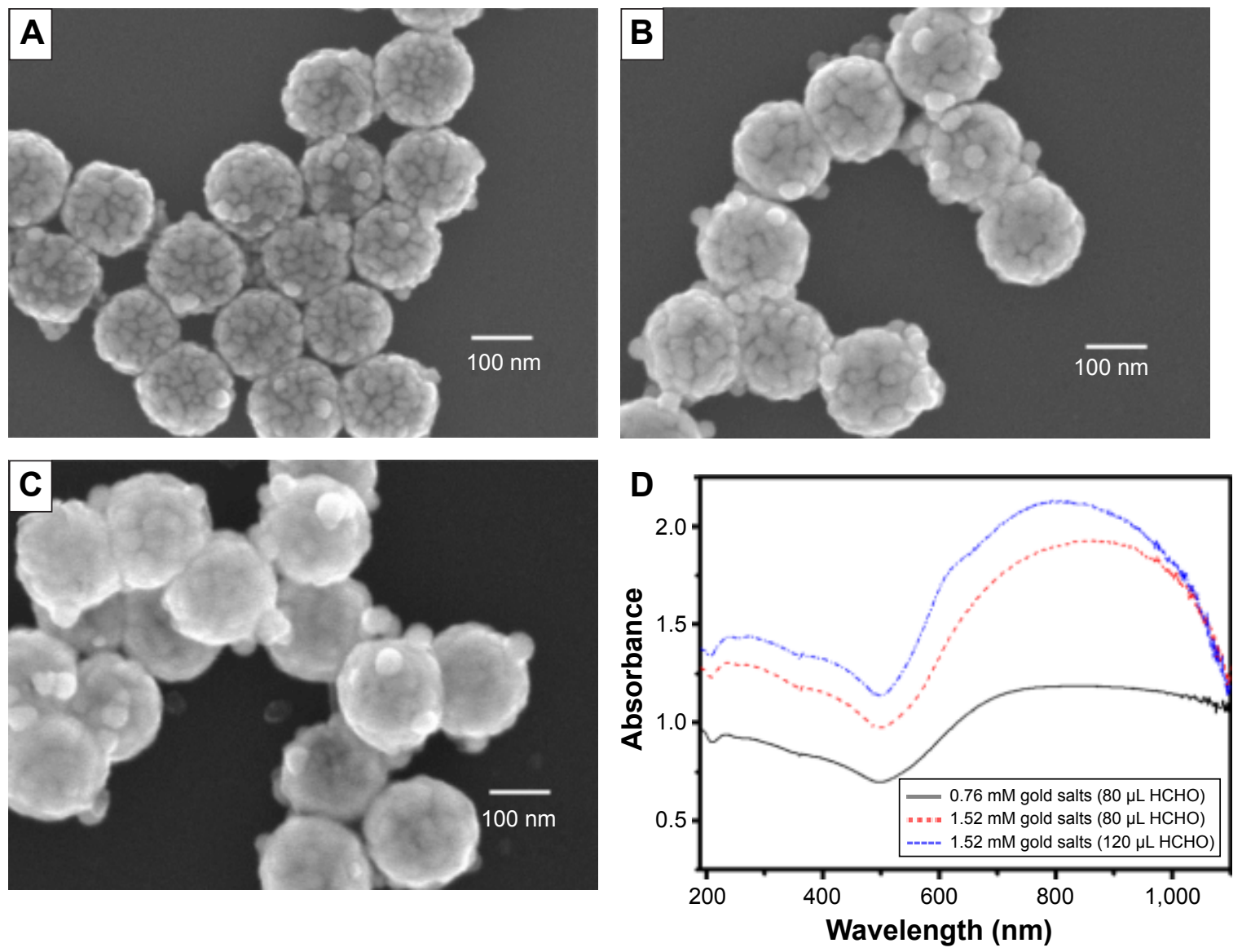

Figure SI SEM images of SGNS (Silica core dia. $=120 \mathrm{~nm}$ ) prepared by adding different gold salts in the presence of $\mathrm{HCHO}$ reducing agent $(80-120 \mu \mathrm{L})$ : $(\mathbf{A}) 0.76 \mathrm{mM}$ gold salts and $80 \mu \mathrm{L} \mathrm{HCHO}$ (Au shell thickness $=20 \mathrm{~nm}$ ), (B) I.52 mM gold salts and $80 \mu \mathrm{L} \mathrm{HCHO} \mathrm{(Au} \mathrm{shell} \mathrm{thickness}=28 \mathrm{~nm}$ ), (C) I.52 mM gold salts and I $20 \mu \mathrm{L} \mathrm{HCHO}$ (Au shell thickness $=35 \mathrm{~nm}$ ), and (D) UV-Vis spectra of SGNS prepared by different concentrations of gold salts that are corresponding to the SEM images of (A), (B), and (C), respectively.

Abbreviations: SGNS, silica-gold nanoshell; UV-Vis, UV-visible spectroscopy; SEM, scanning electron microscopy; dia., diameter.

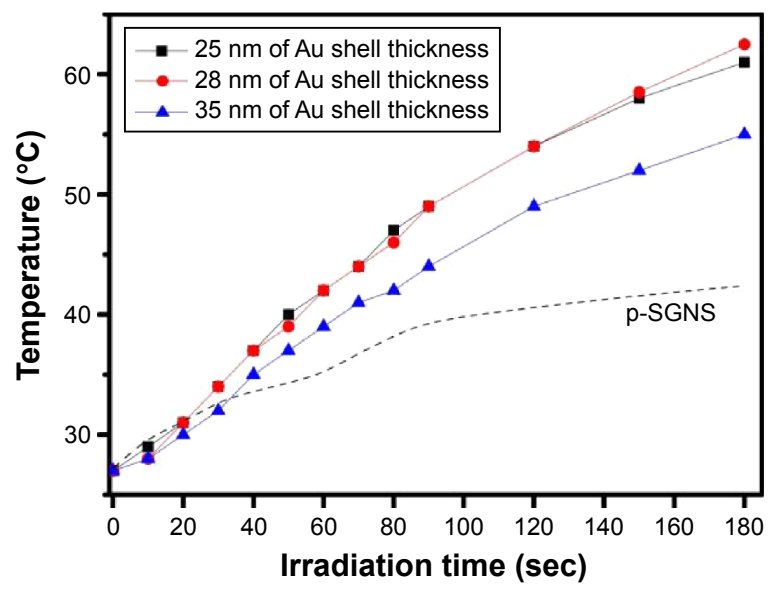

Figure S2 Temperature increment of SGNS solution with the increase of exposure times of NIR light $\left(820 \mathrm{~nm}, 30 \mathrm{~W} / \mathrm{cm}^{2}\right)$. As-prepared samples $($ silica core $=120 \mathrm{~nm})$ exhibited the different thickness of the gold layer. In reference, the temperature increment by p-SGNS was provided for the comparison.

Abbreviations: SGNS, silica-gold nanoshell; p-SGNS, partially covered-silica-gold nanoshell; NIR, near infra-red. 
International Journal of Nanomedicine

Dovepress

\section{Publish your work in this journal}

The International Journal of Nanomedicine is an international, peerreviewed journal focusing on the application of nanotechnology in diagnostics, therapeutics, and drug delivery systems throughout the biomedical field. This journal is indexed on PubMed Central, MedLine, CAS, SciSearch ${ }^{\circledR}$, Current Contents ${ }^{\circledR} /$ Clinical Medicine,
Journal Citation Reports/Science Edition, EMBase, Scopus and the Elsevier Bibliographic databases. The manuscript management system is completely online and includes a very quick and fair peer-review system, which is all easy to use. Visit http://www.dovepress.com/ testimonials.php to read real quotes from published authors.

Submit your manuscript here: http://www.dovepress.com/international-journal-of-nanomedicine-journal 